\title{
INTEGRATION OF WEATHER AVOIDANCE AND TRAFFIC SEPARATION
}

\author{
Maria C. Consiglio, James P. Chamberlain, and Dr. Sara R. Wilson \\ NASA Langley Research Center \\ Hampton, Virginia
}

\begin{abstract}
This paper describes a dynamic convective weather avoidance concept that compensates for weather motion uncertainties; the integration of this weather avoidance concept into a prototype 4-D trajectory-based Airborne Separation Assurance System (ASAS) application; and test results from a batch (non-piloted) simulation of the integrated application with high traffic densities and a dynamic convective weather model. The weather model can simulate a number of pseudo-random hazardous weather patterns, such as slow- or fast-moving cells and opening or closing weather gaps, and also allows for modeling of onboard weather radar limitations in range and azimuth. The weather avoidance concept employs nested "core" and "avoid" polygons around convective weather cells, and the simulations assess the effectiveness of various avoid polygon sizes in the presence of different weather patterns, using traffic scenarios representing approximately two times the current traffic density in en-route airspace.
\end{abstract}

Results from the simulation experiment show that the weather avoidance concept is effective over a wide range of weather patterns and cell speeds. Avoid polygons that are only 2-3 miles larger than their core polygons are sufficient to account for weather uncertainties in almost all cases, and traffic separation performance does not appear to degrade with the addition of weather polygon avoidance. Additional "lessons learned" from the batch simulation study are discussed in the paper, along with insights for improving the weather avoidance concept.

\section{Introduction}

Weather presents a significant source of uncertainty and potential delay for any air traffic management (ATM) system or concept, and convective weather (thunderstorm activity) presents particular challenges. Convective weather is challenging for flight and for ATM systems because it can include rapidly changing weather conditions, heavy rain, severe to extreme turbulence, high winds and gusts, hail, icing, lightning, severe downdrafts and microbursts, reduced ceiling and visibility, and instrument meteorological conditions (IMC) [1]. Flight planners and traffic flow management personnel will strategically avoid flight into areas with substantial coverage of current or forecast convective activity when possible, but there are still many occasions in normal operations when tactical avoidance of convective weather cells is required. In these cases flight crews will consider multiple information sources but will typically use onboard weather radar as their primary information source for the location of cells with the most intense precipitation echoes, and then employ guidelines or "rules of thumb" to determine safe avoidance distances from these echoes and their associated hazards. These guidelines vary with company policy and with flight crew judgment, but a typical example is the FAA guideline to avoid by at least 20 miles any thunderstorm identified as severe or giving an intense radar echo [2]. A flight crew might also modify the distance by which it avoids an onboard weather radar echo based on other factors such as pilot reports, visual cues, local knowledge, prior experience, and winds aloft.

Dynamic convective weather avoidance is especially challenging for emerging en-route 4-D trajectory-based ATM concepts because of the unpredictability of weather cell "morphing" (or shape-changing) and translation [3] - thunderstorms evolve in an unpredictable manner and the future "velocity vector" of any given cell edge is generally unknown to any degree of precision. Furthermore, the weather detection and prediction capabilities of both airborne and ground-based weather equipment are limited; for example, onboard weather radar shows current echoes but is limited in range and azimuth. Trajectory-based ATM applications must compensate for these weather uncertainties and equipment limitations when building trajectories that avoid thunderstorms as well as traffic conflicts. For example, an ATM application may be given a defined 
"fly no closer than" distance for weather cells of a particular intensity (provided by guidelines and/or crew input), but if its trajectories are to reliably meet this constraint it must compensate for future unpredictable movement of the cells.

The weather avoidance concept described in this paper assumes an ability to define weather "core" polygons laterally around detected hazardous weather areas (including any specified "fly no closer than" safety distances), and then to construct slightly-larger "avoid" polygons around the core polygons to account for weather movement uncertainty. There are numerous techniques in the public domain that could be employed to build these core polygons using radar imagery and safety distance parameters as input; for example, a previous study included both models and applications of weather polygons and pilot preferences when choosing weather avoidance routes [3]. The experiment described in this paper used a simple weather cell model and built core polygons at a fixed 20 nautical mile (NM) distance from the weather cell edges to test the weather avoidance concept. Weather cell morphing and translation were modeled by pseudo-randomly morphing the surrounding core polygon's vertex positions and by translating the polygon at a specified velocity, respectively (weather cell growth/decay could be similarly modeled but was not examined in this study). Avoid polygons were built at a defined distance around each of the core polygons; this distance was an independent variable in the study of the concept's effectiveness. In all cases the polygon dimensions were uniform at all altitudes - that is, they were "extruded" vertically through all altitudes in the airspace of interest - but the concept could be generalized to weather polygons that vary in dimension and extent with altitude.

The weather avoidance concept has been integrated into NASA Langley Research Center's Autonomous Operations Planner (AOP), which is a prototype airborne separation assurance system (ASAS) ATM application [4,5], but the concept could also be applied to a ground-based ATM application [6,7]. The AOP is designed to detect traffic conflicts using ADS-B state and intent information, and weather conflicts using the avoidance concept and input from an onboard weather radar model, and then to provide crews with conflict-free trajectories that simultaneously avoid both traffic and weather conflicts. The resolution trajectories generated by AOP satisfy traffic flow management constraints such as a required time of arrival (RTA) at a metering fix.

The next section of the paper describes the weather avoidance concept in more detail, followed by a section describing its integration into an ASAS application (AOP). Next, our weather model for simulating weather cell patterns, onboard weather radar and polygon generation is described, followed by a section that describes a batch (non-piloted) simulation experiment we conducted that used the weather model to test the integrated weather avoidance and traffic separation (ASAS) application. Subsequent sections present the results of the experiment, discuss the results with respect to concept effectiveness and possibilities for improvement, and provide some concluding remarks.

\section{The Weather Avoidance Concept}

As stated in the introduction, the weather avoidance concept assumes the availability of an external "polygon generator" application that is capable of constructing core polygons around detected convective weather cells and any specified minimum safety distance that is to be maintained from them. It is also assumed that the polygon generator would provide a new, updated set of core polygons at regular intervals as new weather cell data are detected. These core polygons would then become "hard constraints" for an ATM trajectorygeneration algorithm, in the same way that restricted airspace or the minimum separation distance around other aircraft would also be hard constraints; that is, conflict-free 4-D trajectories must avoid all hard constraints. However, the weather core polygons differ from the other hard constraints in that their positions, velocities, shapes, and even the total number of them change with their updates over time in unpredictable ways. This unpredictability tends to make the computation of weather-conflict-free 4-D trajectories an intractable problem - there is no way to prevent an updated core polygon edge from morphing or moving over the aircraft's position on a formerly conflict-free trajectory that was skirting the polygon's old position.

One approach to solving this problem is to construct a slightly-larger avoid polygon of size " $\mathrm{D}$ " around each core polygon (illustrated notionally in 
Figure 1) and to define these avoid polygons as "soft constraints." If possible, soft constraints will be avoided along with hard constraints when (new) weather data are received and trajectories are (re)computed, but if no such solutions are available then a trajectory may be offered that penetrates a soft constraint (with minimal-penetration solutions given preference by the algorithm over progressively deeper penetrations). More significantly, if a formerly conflict-free trajectory later becomes conflicted with an avoid polygon that has moved over the aircraft's current position, the current trajectory is still acceptable (all hard constraints are still avoided), but the algorithm will begin seeking a new trajectory that will exit the avoid polygon and is subsequently free of conflicts with all hard and soft constraints (Figure 2). In essence, the avoid polygons buy the algorithm time and give it early warning that one or more core polygon edges are moving toward its trajectory.

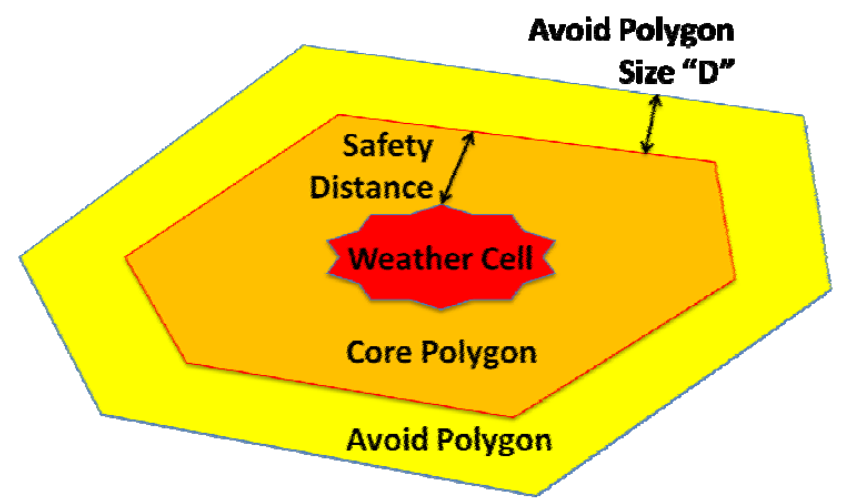

Figure 1. Weather Core and Avoid Polygon

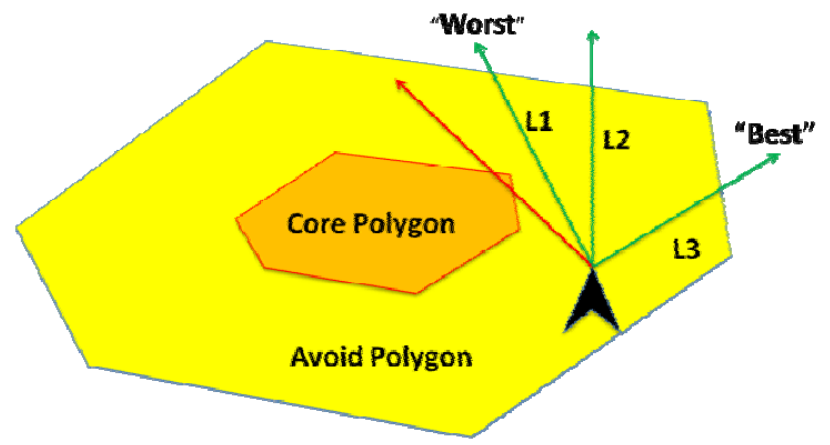

Figure 2. Weather Avoidance Implementation

Many variables can be used and assigned different weights by an algorithm when computing trajectories that must pass through avoid polygons; such trajectories may be required either to clear other hard constraints or to exit an avoid polygon that has moved over the aircraft's position. Some examples of these variables include distance from core polygons, time or distance spent within avoid polygons, time/distance spent within overlapping avoid polygons, and aggressiveness of exit maneuver relative to current heading. To date we have selected variables and weightings that maximize distances from core polygons, and minimize time flown in avoid polygons, when computing trajectories that pass through or exit avoid polygons. Figure 2 (not drawn to scale) shows the effects of using these variables when computing an exit trajectory from an avoid polygon: the red trajectory is unacceptable because it penetrates the core polygon within the trajectory's look-ahead time (represented by the arrow lengths), and the green trajectory labeled "L3" is optimal because it maximizes distance from the core polygon and exits the avoid polygon in minimum time. Green trajectories L2 and L1 are acceptable but progressively less desirable (i.e., they are lower-weighted as solutions) in this example with only a single core and avoid polygon; currently there is no penalty for maneuver aggressiveness when exiting an avoid polygon, other than a maximum heading change of 60 degrees. Figure 3 introduces a second, overlapping core and avoid polygon to the example shown in Figure 2. In this case trajectory L2 is optimal in meeting all of the weighted variables; it "splits the difference" between the two core polygons and minimizes total time in avoid polygons (time that would be spent in overlapping avoid polygon areas is doubly penalized).

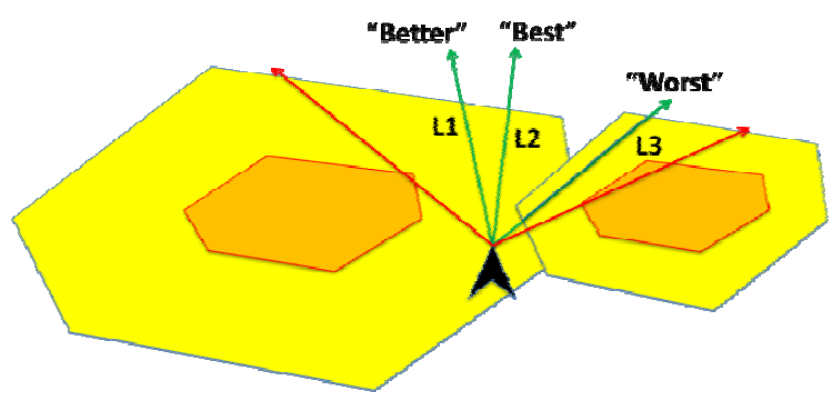

Figure 3. Weather Trajectory Selection

Clearly, large avoid polygons (i.e., large values of " $D$ " in Figure 1) are likely to minimize core polygon penetrations but at the same time block large areas of airspace and introduce larger system 
disruptions. Small avoid polygons are likely to improve trajectory and airspace efficiency but be less effective in preventing core polygon intrusions. The optimal avoid polygon size range would likely be affected by the maximum cell edge speeds and the types of weather patterns, and should also be affected by the update frequency for new core polygons (i.e., more frequent updates should allow for smaller avoid polygons). The avoid polygon size and weather pattern type/speed are the independent variables in the experiment described later in the paper.

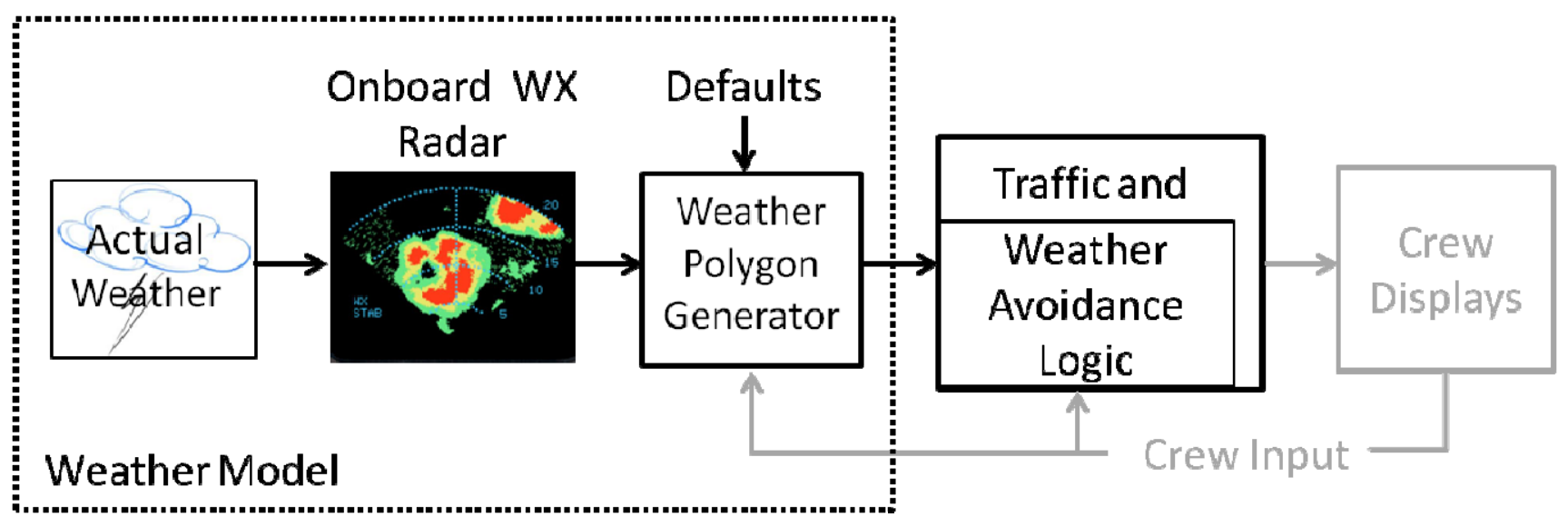

Figure 4. On-board Weather Avoidance Architecture

\section{Integrating Weather Avoidance into an ASAS Application}

Figure 4 depicts a notional architecture showing the main components of an ASAS application (i.e., AOP) integrated with the weather avoidance concept and using onboard weather radar as its hazardous weather information source. Onboard weather radar was selected for this architecture because: 1) it is currently available and required on all transport category aircraft in air transportation service, 2) it is the primary weather information source for tactical avoidance of convective weather when in IMC, 3) it provides the most immediate and frequently-updated image of hazardous weather relevant to the aircraft's flight path, and 4) its status as an onboard weather information source is more consistent with the autonomous nature of an ASAS application than critical reliance on reception of ground-based weather information sources. That is, while the future availability of flight information services (FIS) weather data may augment and improve strategic, and possibly tactical, weather avoidance trajectory generation by an ASAS application, it should not be relied upon as the primary weather information source.
As depicted in Figure 4, the onboard weather radar would provide a near-real-time weather image to a polygon generator application; this weather image would necessarily be limited or "cropped" by the radar's azimuth and range limitations. The polygon generator would compute a set of core polygons around weather in this image that exceeds a specified intensity level, using either default safety distance settings and/or input from the flight crew, and also generate a set of avoid polygons surrounding the core polygons. This set of core and avoid polygons would then be provided to the weather avoidance logic embedded in the ASAS application, to be used as hard and soft constraints when computing conflict-free trajectories. At regular intervals (30 seconds in the current simulation of this architecture), the polygon generator would provide an updated set of core and avoid polygons to the weather logic, at which time the logic would discard the old set and replace it with the new, and reassess the current trajectory for conflicts. The integrated weather avoidance/ASAS application would present the weather imagery and polygons, trajectories and weather/traffic conflicts (if any) to the flight crew on the appropriate displays, which should facilitate crew decisions and possible input to the polygon generator and/or weather avoidance logic. 
In order to test the effectiveness of the integrated weather/ASAS application in the batch simulation described later in the paper, the actual weather, onboard weather radar and polygon generator was represented with a simple parametric convective weather model, as shown by the dashed outline in Figure 4 and as described in the next section. Also, the current instantiation of the architecture shows only the weather core and avoid polygons (and not the actual weather imagery) on each simulated aircraft's navigation display, since display of the actual weather imagery is not needed for batch simulations with automated pilot models in place of human pilots.

\section{The Weather Model}

The weather model is designed to simulate a number of pseudo-random convective weather patterns, such as slow- or fast-moving cells and opening or closing weather gaps, and to generate the core and avoid weather polygons that would result from an aircraft's onboard radar encountering this simulated weather from a given position. It is highly parameterized, enables modeling of onboard weather radar limitations in range and azimuth, and dynamically updates the polygons as the weather moves and the onboard weather radar view changes.

The weather model accepts as input a cell pattern and safety distance specified by the researcher, and builds simple core polygons around these cells at that distance. Each cell is assumed to have the same cross-section at all altitudes of interest, so the resulting polygons are "extruded" vertically throughout all altitudes in the test area. For the experiment described in this paper, all patterns were built up from one or two $10 \mathrm{NM}$-diameter circular weather cells with uniform $20 \mathrm{NM}$ safety distances around them, which resulted in $50 \mathrm{NM}$ circumscribed-diameter core polygons (hexagons) around each of these cells.

Each of the weather cells in a pattern are assumed to randomly morph (that is, change shape by dilating or contracting along any given edge), and each cell may also be assigned a translation velocity. Cell translation is simulated by updating each of its core polygon vertex positions at the specified translation rate. Cell morphing is simulated by randomly repositioning the core polygon vertices around their nominal locations at user specified update rates and distances. For the experiment described here, each vertex position was randomly updated within $\pm 3 \mathrm{NM}$ of its nominal position on a 5-minute schedule, with intermediate vertex positions interpolated in time. This results in polygon vertices and edges that randomly morph at rates varying from 0 to 70 knots (kts), superimposed on any overall cell translation velocity.

The weather model provides these dynamicallyupdating "scenario-wide" core polygons to all aircraft in a simulation scenario at a regular update rate (30 seconds for the experiment described here), but must also model for each aircraft the unique core and avoid polygons that it would generate based on its radar view of the weather cells associated with these scenario-wide polygons. The weather model does this for each aircraft by employing a simple cropping algorithm to simulate the radar's limited range and azimuth, as shown in Figure 5. In this figure the scenario-wide core polygon is shown in tan, the radar-cropped core polygon detected by this aircraft is orange, and the avoid polygon it would build around this cropped core polygon is outlined in green. For this experiment the radar cropping range was set at $80 \mathrm{NM}$ (equivalent to detecting the actual weather cell at $100 \mathrm{NM}$, due to the $20 \mathrm{NM}$ safety distance) and the azimuth was cropped at \pm 60 degrees.

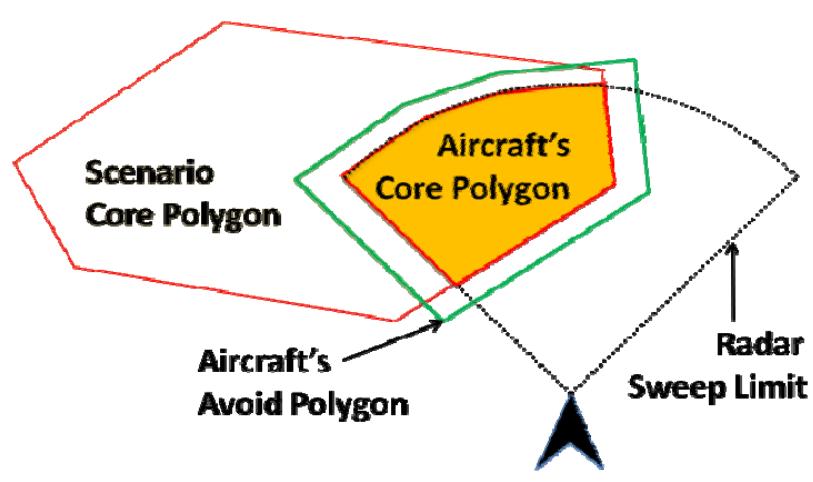

Figure 5. Weather Radar Simulation

\section{Experiment Description}

An exploratory batch simulation study was conducted to investigate the effectiveness of the integrated weather/ASAS application in the presence of various weather patterns and with various avoid polygon sizes, using traffic scenarios that represent 
approximately twice the current traffic density in enroute airspace. Five different weather patterns were scripted to model a range of complexity levels and are illustrated in Figure 6. The "Stationary Cell" pattern consists of a single weather polygon that is stationary at the test region center, while the " 30 -knot Cell" and "60-knot Cell" patterns consist of a polygon translating across the test region at 30 and 60 kts, respectively. The “Opening Gap” pattern consists of two initially overlapping polygons, one stationary and the other translating away at 30 kts to open an airspace gap in between them. The "Closing Gap" pattern has one stationary polygon and another polygon translating toward it that closes an airspace gap between them, the reverse of the previous case.

\section{Simulation Platform}

The simulation runs described herein were conducted in the Air Traffic Operations Laboratory (ATOL) at NASA Langley Research Center utilizing a distributed simulation platform known as the Airspace \& Traffic Operations Simulation (ATOS) $[4,5]$. The ATOS batch platform is comprised of hundreds of real-time aircraft simulators, each equipped with a version of AOP in which the new weather avoidance functionality is implemented. Conceptually, the ATOS batch simulation platform is a system composed of multiple distributed autonomous software agents modeling ASASequipped aircraft flown by a pilot model application. In the current study, the pilot model was configured to react to all traffic and weather conflict alerts by requesting a resolution trajectory from the AOP and executing it.

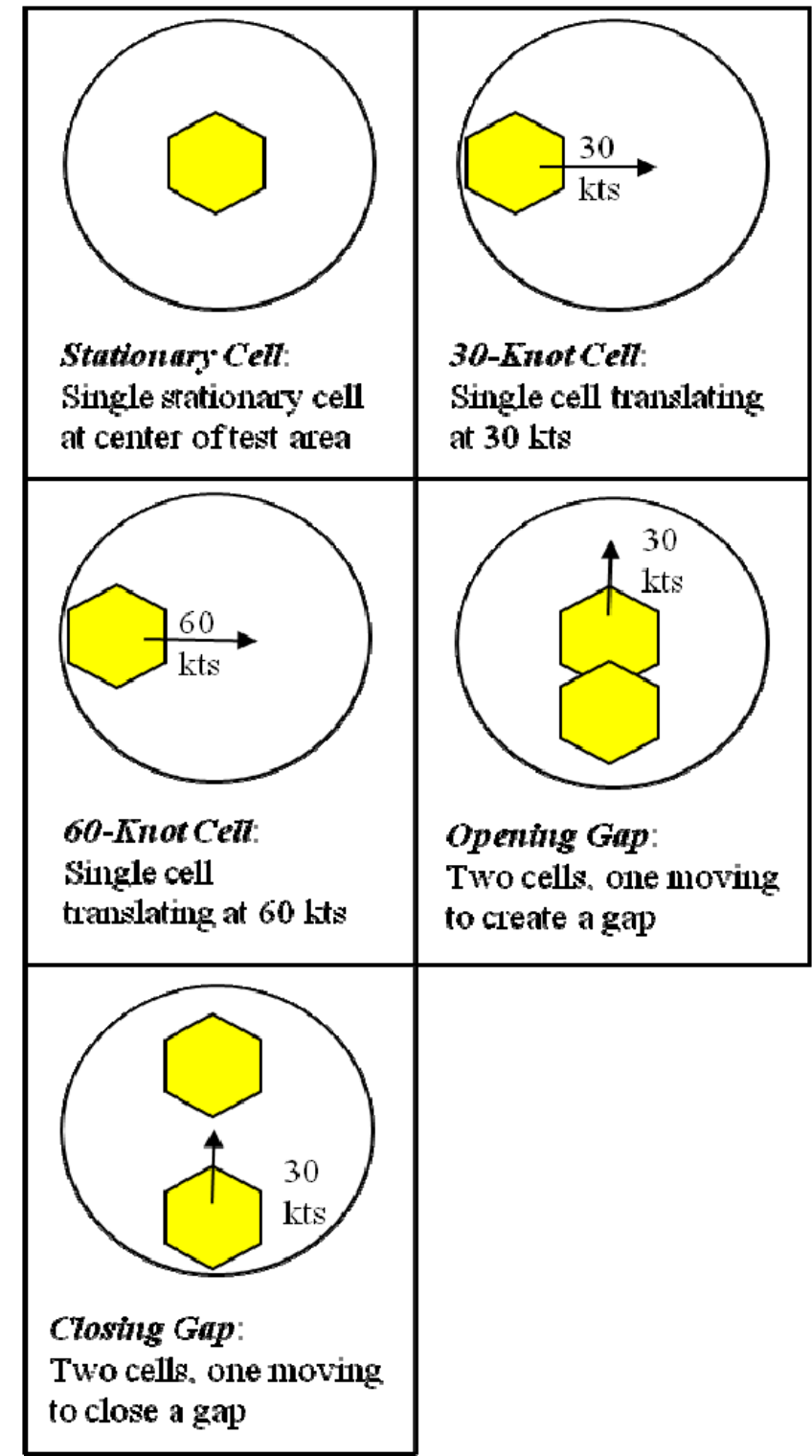

Figure 6. Weather Patterns

\section{Experiment Scenarios and Assumptions}

The experiment scenarios consisted of a notional en route airspace sector modeled as a circular area with a diameter of $160 \mathrm{NM}$. Aircraft were generated at random points along a circle $80 \mathrm{NM}$ (approximately 10 flying minutes) away from the test region boundary, with initially straight trajectories traversing the test region in sets of converging flows towards multiple metering fixes placed approximately $300 \mathrm{NM}$ outside the test region. More information on the random scenario simulation platform can be found in [8]. Each aircraft was assigned an RTA constraint (set to its ETA at 
generation time) at its respective metering fix. All aircraft in the simulation were generated at cardinal altitudes uniformly distributed between Flight Level (FL) 290 and FL 390. ADS-B air-to-air reception was modeled to be perfect (no degradation or errors due to interference) out to a range of $120 \mathrm{NM}$, with no reception beyond this range. A wind field was present, with uniform winds of 40-50 kts at each flight level and moderate changes in speed and direction with altitude, but no wind forecast errors were introduced (i.e, all aircraft had ideal knowledge of the wind field). Weather patterns were extruded vertically, as previously stated, which enforced lateral resolution maneuvers around weather conflicts. Traffic conflicts could be resolved either laterally or vertically.

The traffic density in all scenarios was maintained at a level representative of twice the current traffic density levels for high altitude, enroute airspace sectors in the National Airspace System. Details on the traffic density estimation are contained in [9]. This density equated to 60-70 aircraft concurrently in the $160 \mathrm{NM}$ circular test area throughout each test run of 160 minutes.

\section{Experiment Design}

A 5x6 factorial experiment design was used to collect data on the five weather patterns and six avoid polygon sizes, for a total of 30 experiment scenarios as shown in Table 1 . The six levels of avoid polygon size represent a range from $1 \mathrm{NM}$ to $9 \mathrm{NM}$.

Table 1: Experiment Design Matrix

\begin{tabular}{|c|c|}
\hline Weather Pattern & $\begin{array}{c}\text { Avoid Polygon } \\
\text { Size (NM) }\end{array}$ \\
\hline Stationary Cell & $1,2,3,5,7,9$ \\
\hline 30-Knot Cell & $1,2,3,5,7,9$ \\
\hline 60-Knot Cell & $1,2,3,5,7,9$ \\
\hline Opening Gap & $1,2,3,5,7,9$ \\
\hline Closing Gap & $1,2,3,5,7,9$ \\
\hline
\end{tabular}

Three sets of baseline scenarios were also conducted for each of the five weather patterns. Baseline 1 corresponded to scenarios with both traffic and weather conflict resolutions disabled, and thus no attempts were made to resolve any conflicts. Baseline 2 scenarios incorporated traffic conflict resolutions but no resolutions around weather conflicts. Baseline 3 scenarios included weather avoidance maneuvers (with a $5 \mathrm{NM}$ avoid polygon size) but no traffic conflict resolutions.

\section{Experiment Results}

Two replicates of each of the 30 experiment scenarios and the 15 baseline scenarios described above were conducted. The 90 test runs resulted in a total of approximately 20,000 simulated flight hours, or approximately 450 flight hours for each experiment or baseline scenario.

\section{Dependent Measures}

Several dependent measures were examined as applicable for each experiment and baseline scenario, including the number of detected conflicts with traffic and with weather polygons, the number of aircraft loss of separation (LOS) and weather core area (polygon) penetration (CAP) events, and the excess lateral path length that ensued due to traffic and weather avoidance. Results for each of these dependent measures are described in subsequent subsections.

Results for conflict, LOS, and CAP events have been normalized to the number of respective events per thousand flight hours. While this normalization should help convey the operational significance of the numbers, it should also be noted that these normalized results are extrapolations since only about 450 flight hours were simulated for each experiment scenario. For example, a single event (e.g., a CAP) occurring among all the aircraft flying in a particular experiment scenario will translate into a reported (i.e., extrapolated) result of two events per thousand hours.

In the experiment (i.e., non-baseline) scenarios where both weather and traffic conflict resolution algorithms were active, LOS and CAP events were both relatively rare and of forensic interest, so all such events were manually replayed and analyzed by the researchers. This analysis revealed that slightly over half of the LOS and/or CAP events in the raw data were due to simulation errors rather than to failures of the conflict resolution concepts or implementation, and these "simulation error" LOS and CAP events were excluded from the reported results. The simulation errors were typically rare race conditions and corner-case interactions between the Flight Control Computer (FCC), Flight Management System (FMS) and Pilot Model (PM) code modules. 
PM errors would cause the PM logic to stop responding correctly to the AOP's conflict-resolution alerts and commands, and were detected by the resulting CAP or LOS events. The FCC/FMS errors would cause departures from the commanded (and LOS/CAP-protected) flight path and in some cases would result in extensive circling of the aircraft in an attempt to recapture the commanded flight path. Manual examination of several hundred flight tracks (out of approximately 50,000 total) indicated that this circling simulation error occurred in only a few percent of the sampled flights, but when it did occur it significantly added to the total path length of the flight.

It should be noted that while these simulation errors appear to be relatively rare and were manually excluded from the reported LOS and CAP results, as of this writing there is no practical, automated mechanism to detect and exclude all other such errors from the detected conflict count and excess path length dependent measures. Since the simulation errors appear relatively few compared to the large number of detected conflicts, they are unlikely to dominate the reported results of this dependent measure. Likewise, the errors should not greatly affect the median excess path length dependent measure because of the much larger number of errorfree cases also included in this measure. However, the errors will affect the maximum excess path length and, to some extent, the mean excess path length measures. The effects of the likely presence of simulation errors in the excess path length dependent measures are described in the respective Discussion subsection.

\section{Number of Detected Conflicts}

The number of conflicts was computed as the total number of conflict detections for which the predicted LOS, CAP or Avoid Area Penetration (AAP) occurred partially or completely inside the test region. Figures 7 and 8 present the number of traffic and weather conflict detections per 1,000 flight hours, respectively. It can be seen that both traffic and weather conflict counts are higher for larger avoid polygon sizes and more complex weather patterns. The number of weather conflicts in the scenarios with two weather cells (Opening Gap and Closing Gap) is approximately two times that in the scenarios with only one weather cell (Stationary, 30knot Cell, and 60-knot Cell), as expected.

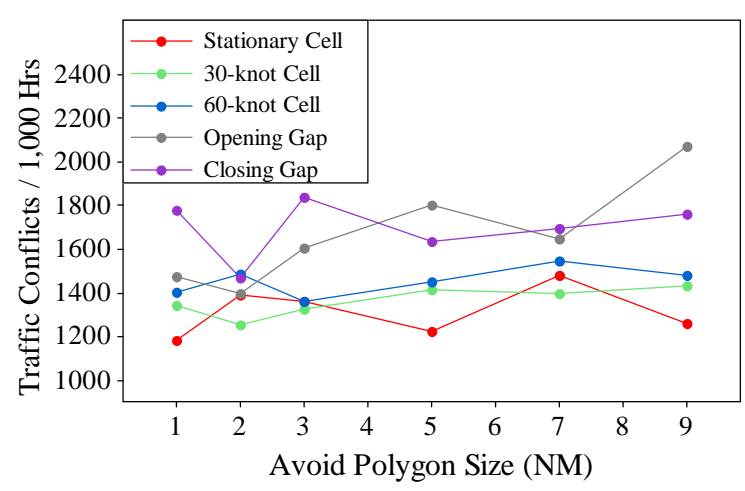

Figure 7. Traffic Conflicts/1000 Hours

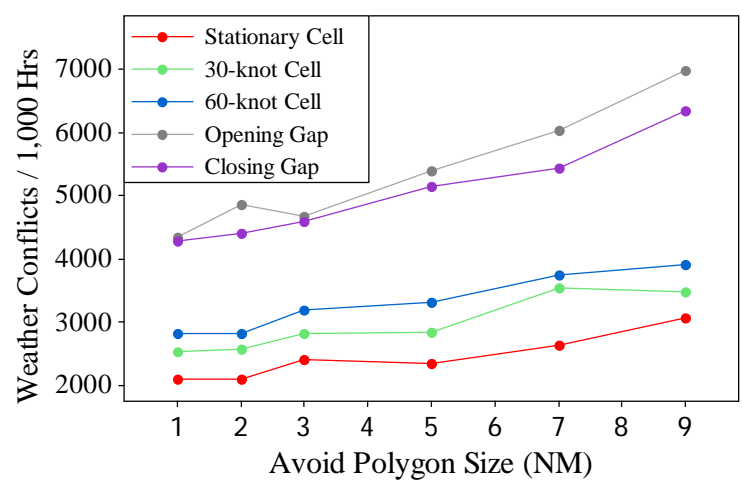

Figure 8. Weather Conflicts/1000 Hours

Statistical analysis of the dataset was performed using an Analysis of Variance (ANOVA). Significant main effects were found for avoid polygon size $(p=$ $0.0071)$ and weather pattern $(p<0.0001)$ for the number of traffic conflict detections. However, the interaction between these two factors was not significant $(p=0.0662)$. Note that a $p$-value $<0.05$ indicates a statistically significant effect. Avoid polygon size $(p<0.0001)$, weather pattern ( $p<$ $0.0001)$ and the interaction $(p<0.0001)$ were all found to have a significant effect on the number of weather conflict detections.

\section{Losses of Separation}

The number of losses of separation per 1,000 flight hours is shown in Table 2 for each scenario. The criteria for LOS were a closest point of approach (CPA) within $5 \mathrm{NM}$ laterally and $800 \mathrm{ft}$ vertically. LOS events occurred in only two of the 30 experiment scenarios, and both weather pattern and avoid polygon size seem to have little impact on this metric. The LOS in the run with the 30-knot cell and an avoid polygon size of $7 \mathrm{NM}$ resulted in a CPA of $2.344 \mathrm{NM}$. In the run with the opening gap weather 
pattern and a $5 \mathrm{NM}$ avoid polygon, the LOS had a CPA of $4.841 \mathrm{NM}$. By comparison, the LOS numbers are quite high (hundreds) for both the Baseline 1 and Baseline 3 scenarios where the traffic resolution logic was disabled. There were no LOS events in the Baseline 2 scenarios where traffic resolution was enabled but weather resolution was disabled.

Table 2. LOS per 1000 Flight Hours

\begin{tabular}{|c|c|c|c|c|c|c|c|c|c|}
\hline \multirow[t]{2}{*}{ Weather Pattern } & \multicolumn{6}{|c|}{ Avoid Polygon Size (NM) } & \multicolumn{3}{|c|}{ Baseline Scenarios } \\
\hline & 9 & 7 & 5 & 3 & 2 & 1 & Base1 & Base2 & Base3 \\
\hline Stationary Cell & 0 & 0 & 0 & 0 & 0 & 0 & 528 & 0 & 614 \\
\hline 30-Knot Cell & 0 & 2 & 0 & 0 & 0 & 0 & 545 & 0 & 569 \\
\hline 60-Knot Cell & 0 & 0 & 0 & 0 & 0 & 0 & 527 & 0 & 660 \\
\hline Opening Gap & 0 & 0 & 2 & 0 & 0 & 0 & 553 & 0 & 764 \\
\hline Closing Gap & 0 & 0 & 0 & 0 & 0 & 0 & 600 & 0 & 698 \\
\hline
\end{tabular}

Table 3. CAP per 1000 Flight Hours

\begin{tabular}{|c|c|c|c|c|c|c|c|c|c|}
\hline \multirow[t]{2}{*}{ Weather Pattern } & \multicolumn{6}{|c|}{ Avoid Polygon Size (NM) } & \multicolumn{3}{|c|}{ Baseline Scenarios } \\
\hline & 9 & 7 & 5 & 3 & 2 & 1 & Base1 & Base2 & Base3 \\
\hline Stationary Cell & 0 & 0 & 0 & 0 & 4 & 2 & 693 & 646 & 0 \\
\hline 30-Knot Cell & 0 & 0 & 0 & 0 & 0 & 2 & 759 & 708 & 0 \\
\hline 60-Knot Cell & 0 & 0 & 0 & 0 & 0 & 16 & 709 & 646 & 0 \\
\hline Opening Gap & 0 & 0 & 5 & 2 & 2 & 2 & 1326 & 1343 & 0 \\
\hline Closing Gap & 2 & 7 & 2 & 9 & 5 & 14 & 1373 & 1347 & 7 \\
\hline
\end{tabular}

\section{Weather Penetrations}

Table 3 presents the number of CAP events per 1,000 flight hours for each scenario. The highest number of CAP events in the experiment scenarios occurred with the 60-knot Cell and Closing Gap weather patterns and the smallest avoid polygon size of $1 \mathrm{NM}$, but even in these two cases the CAP counts are less than 20 per 1,000 flight hours. By comparison, the CAP numbers are quite high (hundreds to thousands) for both the Baseline 1 and Baseline 2 scenarios where the weather resolution logic was disabled. The number of CAP events in the baseline scenarios with two weather cells (Opening Gap and Closing Gap) is approximately two times that in the baseline scenarios with only one weather cell (Stationary, 30-knot Cell, and 60-knot Cell), as expected.

Figure 9 shows the distance the aircraft penetrated the core polygon for each CAP that occurred during the scenario runs. Of the 33 penetrations, 24 were less than $1.5 \mathrm{NM}$ into the core. All of the remaining larger penetrations, including the three largest penetrations of between 9 and
$10 \mathrm{NM}$, occurred with the closing gap weather pattern.

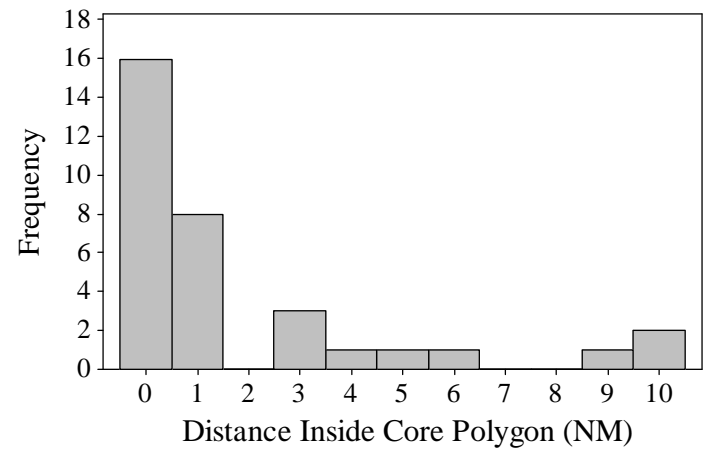

Figure 9. CAP Depth Distribution

\section{Excess Path Length}

Descriptive statistics for excess lateral flight path length by weather pattern and avoid polygon size are shown in Tables 4 and 5, respectively. The Opening Gap and Closing Gap weather patterns result in a mean lateral flight path length almost twice as large as with the Stationary Cell, 30-knot Cell and 60-knot Cell patterns. Additionally, the mean excess 
path length increases slightly for all weather patterns

as the avoid polygon size increases.

Table 4. Excess Path Length (NM) versus Weather Pattern

\begin{tabular}{|l|c|c|c|c|c|c|}
\hline $\begin{array}{c}\text { Weather } \\
\text { Pattern }\end{array}$ & N & Mean & $\begin{array}{c}\text { Std. } \\
\text { Dev. }\end{array}$ & Min. & Median & Max. \\
\hline Stationary Cell & 6461 & 4.3 & 8.1 & 0 & 0.7 & 78 \\
\hline 30-Knot Cell & 6425 & 4.9 & 9.4 & 0 & 0.8 & 116 \\
\hline 60-Knot Cell & 6476 & 5.4 & 10.8 & 0 & 0.8 & 130 \\
\hline Opening Gap & 6497 & 8.9 & 14.1 & 0 & 3.2 & 358 \\
\hline Closing Gap & 6438 & 9.1 & 14.3 & 0 & 2.9 & 114 \\
\hline
\end{tabular}

Table 5. Excess Path Length (NM) versus Avoid Polygon Size

\begin{tabular}{|c|c|c|c|c|c|c|}
\hline $\begin{array}{c}\text { Avoid Polygon } \\
\text { Size (NM) }\end{array}$ & $\mathbf{N}$ & Mean & $\begin{array}{c}\text { Std. } \\
\text { Dev. }\end{array}$ & Min. & Median & Max. \\
\hline 1 & 5333 & 5.4 & 10.2 & 0 & 0.9 & 114 \\
\hline 2 & 5348 & 5.5 & 10.2 & 0 & 0.9 & 108 \\
\hline 3 & 5411 & 6.1 & 11.3 & 0 & 0.9 & 116 \\
\hline 5 & 5392 & 6.7 & 12.7 & 0 & 1.1 & 358 \\
\hline 7 & 5416 & 7.3 & 12.3 & 0 & 1.3 & 130 \\
\hline 9 & 5397 & 8.2 & 13.4 & 0 & 2.0 & 151 \\
\hline
\end{tabular}

Data were averaged over all aircraft in a run, and least squares regression was used to fit the data. The model for excess lateral flight path length is given below. It has an $\mathrm{R}^{2}$ of $96 \%$, indicating that it is a good approximation of the relationship between excess flight path (EP), weather pattern, and avoid polygon size (D).

$$
\begin{array}{ll}
\text { Stationary Cell: } & \text { EP }=3.34+0.22 * D \\
\text { 30-knot Cell: } & \text { EP }=3.93+0.22 * D \\
\text { 60-knot Cell: } & \text { EP }=4.53+0.19 * D \\
\text { Opening Gap: } & \text { EP }=6.13+0.61 * \mathrm{D} \\
\text { Closing Gap: } & \text { EP }=6.71+0.54 * D
\end{array}
$$

Avoid polygon size $(p<0.0001)$, weather pattern $(p<0.0001)$, and the interaction between these two factors $(p<0.0001)$ were found to have a statistically significant effect on the mean excess lateral flight path length.

\section{Discussion of Results}

\section{Number of Detected Conflicts}

Figures 7 and 8 show, respectively, that on average an aircraft flying in these experiment scenarios might expect to encounter between one and two traffic conflicts per hour, and between two and seven weather conflicts per hour. Moreover, Figure 8 shows that the most weather conflicts occur when two weather cells are present and the avoid polygon sizes are large. The increase in conflicts between the one-cell and two-cell scenarios is likely due to twice as much usable airspace being consumed by two weather polygons as by one, and for the same reason the conflict count also increases as the avoid polygon size is increased. That is, the use of large avoid polygons exacts a penalty by increasing the number and rate of weather conflicts that must be resolved by the automation and flight crew.

Operationally, resolving between two and seven weather conflicts per hour should not pose an undue burden on flight crews, but these average numbers tell only part of the story. By detailed inspection of 
the simulation data it is clear that many aircraft negotiate the weather they encounter with the use of only a single conflict resolution, or in some cases with no resolution at all if weather and traffic are not encountered on their original flight plan route. Other aircraft are not so lucky, and some of the outlier cases exceed 30 conflicts per hour, which would almost certainly pose an unacceptable workload to a busy flight crew negotiating through weather cells. Inspection and replay of these outlier flights indicate at least two improvements that could be made to the weather avoidance concept; these improvements are described in the next two paragraphs.

The first improvement to the concept would be to include a "resolution polygon" around each core/avoid polygon set that is slightly larger (e.g., 1$2 \mathrm{NM}$ ) than the avoid polygon. Then when a conflict is detected with a weather core and/or avoid polygon, the resolution logic would attempt to compute a conflict-free trajectory that also clears the resolution polygon. As the core/avoid polygon edges subsequently move with time, this new trajectory should remain conflict-free longer than an optimized trajectory which is computed right up against the edge of an avoid polygon, and which becomes almost immediately conflicted again if/when the avoid polygon moves over it. This phenomenon of repeated new weather conflicts was observed in many of the high-conflict outlier cases, especially when flying resolution trajectories that skirt the downwind side of moving cells (recall that the weather avoidance algorithm assumes no reliable knowledge of cell motion), but also when skirting unpredictably morphing polygon boundaries.

The second concept improvement would be to add a "short-term weather memory" capability to the weather polygon generation software. The concept as it is currently instantiated and modeled assumes that the polygon generation software would use the onboard weather radar imagery as source data, would generate a current set of weather core and avoid polygons from this imagery at frequent intervals, and at each interval would replace the old set of polygons with the new for use by the weather polygon conflict resolution logic. A problem with this concept is that cells to the left and right of the aircraft are cropped and "disappear" at the azimuth limits of the radar as the aircraft moves ahead, and their respective polygons are cropped as well. While a crewmember would remember that a hazardous cell is still abeam the aircraft, even though no longer shown on the radar, the conflict resolution logic has no such shortterm memory and will often propose new trajectories through what it assumes is now weather-free airspace to the left or right of the aircraft. This problem is particularly troublesome when emerging from a close gap between two core polygons but still within both associated avoid polygons - as either the right or left cell disappears from the radar view, the polygon generator eliminates its respective core and avoid polygons, and the conflict resolution logic then commands a somewhat aggressive turn away from the remaining cell to fly clear of its avoid polygon. Needless to say, shortly after commencing this turn the radar "rediscovers" the cell in the direction of the turn, and "forgets" the other cell as it leaves the radar view, resulting in an unnecessary weather conflict and a commanded turn in the opposite direction. This behavior was nicknamed the "windshield wiper instability" because it would often lead to multiple weather conflicts and left-right turns until well clear of both cells. Such behavior and its excessive weather conflicts could be eliminated with a shortterm weather memory built into the polygon generator, where weather polygon segments that move out of radar azimuth limits are retained for one or two minutes and supplied to the resolution logic as part of the current polygon set.

\section{LOS and CAP Events}

As stated in the Results section, only two LOS events occurred in all of the experiment and baseline scenarios where traffic conflict resolution logic was enabled. In both of these cases the logic failed to detect and present the traffic conflict, for reasons that are still under investigation, but neither the weather scenarios, avoid polygon size, nor weather avoidance logic appear to be a factor in these two LOS events. More generally, the addition of weather conflict resolution logic does not appear to affect or compromise the traffic conflict resolution capabilities in the software; LOS rates remain low and do not appear to degrade with the addition of weather polygons and conflict resolutions.

Weather CAP rates are generally quite low: the overall CAP rate for all scenarios where the weather conflict avoidance logic is enabled is approximately $0.6 \%$ of the CAP rate for the Baseline 1 and 2 scenarios where the weather conflict resolution logic 
is disabled. In fact, no CAP events occurred in a majority (20 of 35) of the scenarios where the weather resolution logic was enabled. Based on both the data and playback observations it appears that avoid polygon sizes as small as 2 or $3 \mathrm{NM}$ are effective at preventing CAP events, but an avoid polygon size of $1 \mathrm{NM}$ is too small to prevent some CAP events for the weather patterns evaluated in this experiment, especially for fast-moving cells or closing gaps.

The Closing Gap weather pattern, and to a lesser extent the Opening Gap pattern, generally had more CAP events than the other patterns even with larger avoid polygons. The majority of these CAP events involved only slight "grazing" penetrations into the core polygon, but some resulted in significant penetrations of as much as $10 \mathrm{NM}$. These and other CAP events were examined and analyzed for cause, as described in the following paragraphs.

One reason that more CAP events occur for the two-polygon patterns is because the maneuvers necessary to deviate around these weather areas are sometimes larger than the limits currently built into the conflict resolution logic. The current limits for turn angle, lateral offset distance, etc. are designed for traffic conflict resolutions and are relatively modest compared to what is sometimes required to tactically deviate around weather once it is detected. In cases where a weather conflict could not be resolved because of these limits, the logic would present the conflict but provide no resolution, and the Pilot Model would take no action and continue on the aircraft's original FMS flight plan, sometimes resulting in significant penetrations into the core polygon. It should be possible to eliminate such CAP cases by relaxing the resolution angle and offset limits for weather conflicts.

A related but more serious reason for occasional deep CAP events arises when the gap between polygons is open but narrow and several co-altitude aircraft are concurrently attempting to pass through it. The current resolution logic will seek either a lateral or a vertical resolution but not a composite lateral and vertical resolution, and several CAP events were observed where the logic could not find a resolution through the gap that was free of both traffic and weather conflicts. In these cases the conflicts were presented but a resolution was not provided, resulting in (sometimes significant) CAP events. Some of these CAP events could likely be prevented by adding composite lateral/vertical resolution patterns to the logic, so that aircraft could avoid the weather laterally and each other vertically. However, the more general solution to this airborne separation problem likely involves external means of either reducing traffic/weather complexity so that a conflict-free resolution can always be found, or devising algorithms and/or procedures that provide alternatives when a resolution is not forthcoming. Such a general solution is beyond the scope of this study.

The majority of CAP events were slight penetrations into the core polygon and occurred with a $1 \mathrm{NM}$ avoid polygon size and either the 60-knot Cell or Closing Gap weather patterns. In the case of the 60-knot Cell weather pattern, a CAP would typically occur while an aircraft was closely deviating around the downwind side of the cell and simply got "run over" by the core polygon. With the Closing Gap pattern the aircraft was often "pinched by the gap" - that is, the gap closed after the aircraft was committed to passing through it, resulting in grazing penetrations into one, or sometimes both, of the core polygons on either side of the closing gap. Avoid polygon sizes greater than $1 \mathrm{NM}$ eliminated these grazing CAP penetrations for both the 60-knot Cell and Closing Gap patterns.

A few CAP events were due to software failures in the weather conflict resolution logic, including failure to detect the weather conflict, and turning toward rather than away from a core polygon in the absence of other conflict constraints. The reasons for these software failures are currently under investigation.

\section{Excess Path Length}

The median and mean values of excess path length are relatively low (less than $4 \mathrm{NM}$ and $10 \mathrm{NM}$, respectively) from an operational perspective for all weather patterns and avoid polygon sizes, and are smaller for the simpler weather patterns and smaller avoid polygon sizes, as expected. Such small average excess path lengths should generally not pose a problem for meeting a downstream RTA, since the aircraft could likely compensate for the extra path length with a slight speed increase. However, the maximum excess path length values are high for all experiment scenarios (values between 78 and 
$358 \mathrm{NM}$ ), indicating that some flights are either receiving inefficient routing and/or are "victims" of the simulation errors described in the Results section for path length. We have observed anecdotal evidence of both behaviors. Clearly, such large excess path lengths are unacceptable from an operational perspective, even for a few unlucky aircraft, and these outlier cases are currently under investigation to resolve simulation errors and uncover any inefficient routing issues.

Least squares regression analysis of the excess path length data show longer paths for more complex weather patterns, as expected, but also show a significantly higher penalty (approximately 3 times as great) for larger avoid polygons with the two-cell patterns versus the single-cell patterns. Some of this penalty might be explained because increasing the size of two avoid polygons rather than just one eliminates usable airspace at twice the rate, but some penalty is also likely due to weather "gaps" being eliminated more aggressively, causing more lengthy deviations around both cells. Either way, it highlights the importance of minimizing avoid polygon sizes to maximize efficiency, especially for more complex weather patterns.

\section{Conclusions}

Results from the batch simulation tests of the integrated weather avoidance and traffic separation application described in this paper show that the weather avoidance concept is effective over a wide range of weather patterns and cell speeds. Avoid polygons that are only 2-3 NM larger than their core polygons are sufficient to account for weather uncertainties in almost all cases, and traffic separation performance does not appear to degrade with the addition of weather polygon avoidance.

High conflict counts occurred in some cases due to lack of resolution buffers and/or "short-term weather memory," both of which are discussed in the paper and suggested as improvements to the weather avoidance concept. Weather CAP events occurred in a few cases because no conflict-free resolution could be found, due either to maneuver limits in the resolution algorithms that are insufficient for some weather deviations, or to excessive traffic complexity in narrow weather gaps. Excess path length due to traffic and weather avoidance was small in most cases, but in a few cases was excessive; some of these excessive path length cases were due to simulation errors and some were because the algorithm returned inefficient trajectories. The number of, and causes for, the inefficient trajectories is under investigation.

Future work in this area would include implementing the resolution buffer and weather memory improvements to the weather avoidance concept, and developing and evaluating optimal weather presentations and interfaces for the flight crew. It would also be fruitful to test the integrated weather avoidance and ASAS application with actual recorded weather data and polygon generation software, and to compare the flight tracks and performance from those tests with actual traffic that was flown at the time and in the regions with the recorded weather.

\section{References}

[1] Chamberlain, James and Kara Latorella, Ph.D., 2001, Convective Weather Detection by General Aviation Pilots with Conventional and Data-Linked Graphical Weather Information Sources, $20^{\text {th }}$ Digital Avionics System Conference, Daytona Beach, FL.

[2] Federal Aviation Administration, 2010, Aeronautical Information Manual, 2011 Edition, Newcastle, WA, Aviation Supplies and Academics, Inc., Chapter 7, Section 7-1-29 Thunderstorm Flying.

[3] Rubnich, Mikhail and Rich DeLaura, 2010, An Algorithm to Identify Robust Convective Weather Avoidance Polygons in En Route Airspace, $10^{\text {th }}$ AIAA Conference on Aviation Technology, Integration and Operations, Fort Worth, Texas.

[4] Karr, D., D. Roscoe and R. Vivona, August 2004, An integrated flight-deck decision-support tool in an autonomous flight simulation, AIAA Modeling and Simulation Technologies Conference and Exhibit, AIAA-2004-5261.

[5] Karr, D., D. Roscoe and R. Vivona, August 2006, Conflict detection using variable 4D uncertainty bounds to control missed alerts, AIAA Guidance, Navigation, and Control Conference and Exhibit, AIAA-2006-6057.

[6] McNally, D. and D. Thipphavong, September 2008, Automated separation assurance in the presence of uncertainty, ICAS2008-581, 26th 
International Congress of the Aeronautical Sciences, Anchorage, Alaska.

[7] Matthews, Michael P. and Rich DeLaura, 2010, Assessment and Interpretation of En Route Weather Avoidance Fields from the Convective Weather Avoidance Model, $10^{\text {th }}$ AIAA Conference on Aviation Technology, Integration and Operations, Fort Worth, Texas.

[8] Consiglio et al., 2009, Estimation of Separation Buffers for Wind-Prediction Error in an Airborne Separation Assistance System, Eighth USA/Europe Air Traffic Management Research and Development Seminar (ATM2009).

[9] Wing et al., 2010, Comparison of Airborne and Ground-Based Function Allocation Concepts for
NextGen Using Human-In-The-Loop Simulations, $10^{\text {th }}$ AIAA Conference on Aviation Technology, Integration and Operations, Fort Worth, Texas.

\section{Acknowledgements}

The authors gratefully acknowledge the generous and expert contributions of the many NASA and contractor research and staff members who supported this experiment, and specifically recognize and thank David Wing, Mike Guminsky, Ed Scearce, Jim Sturdy, Bob Vivona, Dave Roscoe, David Karr, John Bunnell, Chris Wyatt, Clay Hubbs, Troy Landers, and Joey Ponthieux.

30th Digital Avionics Systems Conference

October 16-20, 2011 